month 9 (Figure 3); no significant change of RF-IgM was observed (Figure 4).

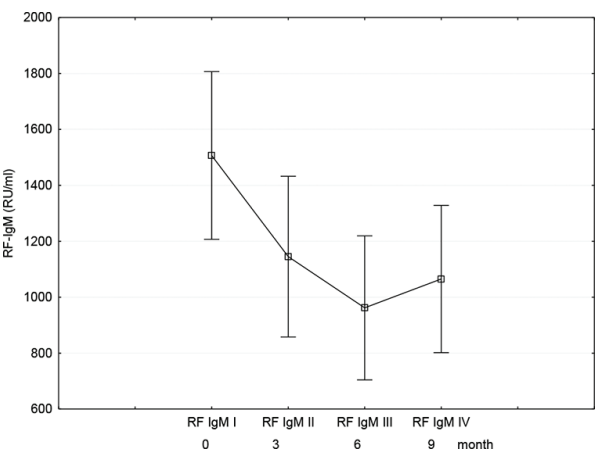

Figure 1. RF-IgM concentrations during RTX treatment.

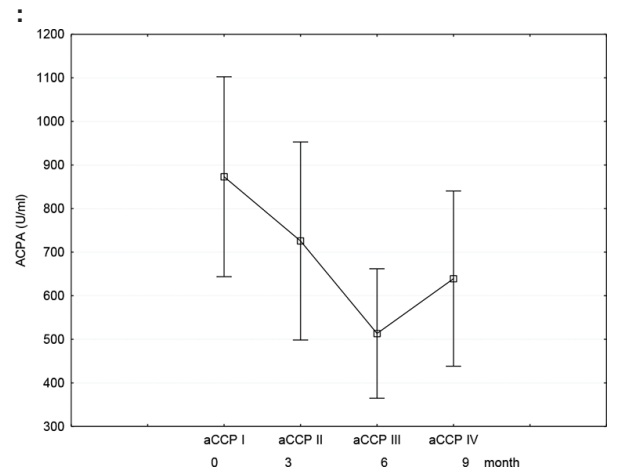

Figure 2. ACPA concentrations during RTX treatment.

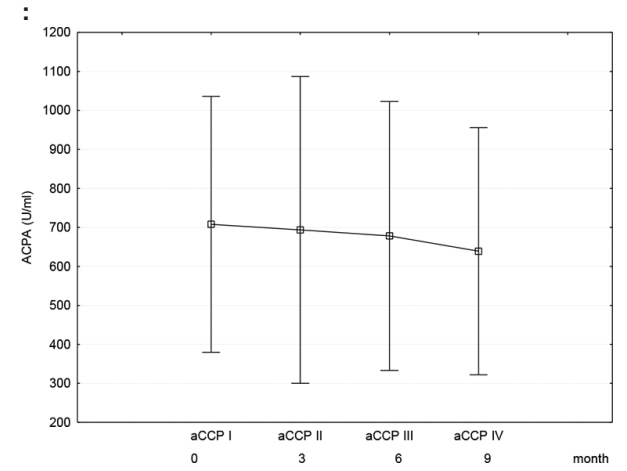

Figure 3. ACPA concentrations during TCZ treatment.

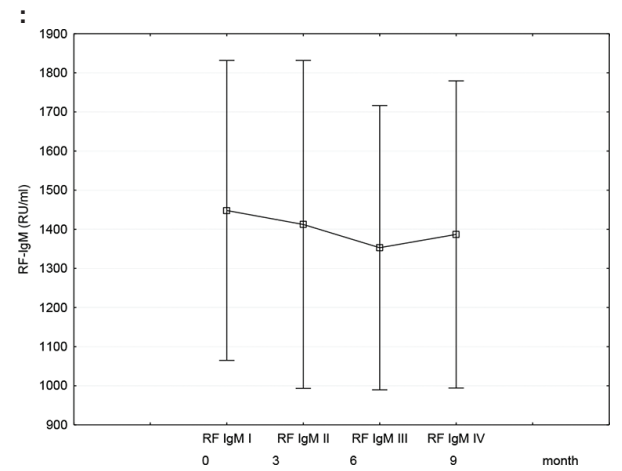

Figure 4. RF-IgM concentrations during TCZ treatment.
Conclusion: The results of the study suggest that both bDMARDs reduced significantly the disease activity in RA patients. The treatment with both drugs was associated with a significant reduction of SAA. However only RTX treatment affected significantly the production of disease specific autoantibodies. Long-term observation is necessary to assess a reliable effect of bDMARDs on the production of marker autoantibodies in association with the disease activity. Disclosure of Interests: Olga Borys: None declared, Bozena Targonska-Stepniak Speakers bureau: Sandoz, Berlin-Chemie, Magdalena Dryglewska: None declared, Maria Majdan Speakers bureau: MSD, UCB, Abbvie, Roche DOI: 10.1136/annrheumdis2019-eular.3476

\section{AB0414 THE IN VITRO EFFECT OF BIOLOGICAL AND CONVENTIONAL DISEASE MODIFYING ANTI RHEUMATIC DRUGS (DMARDS) ON FIBROCYTE DIFFERENTIATION IN RHEUMATOID ARTHRITIS PATIENTS AND HEALTHY CONTROLS}

Helene Tenstad ${ }^{1}$, Pernille Just Vinholt ${ }^{2}$, Christian Nielsen ${ }^{3}$, Hanne Merete Lindegaard ${ }^{1}$, Søren Andreas Just ${ }^{4}{ }^{1}$ Odense University Hospital, Department of Rheumatology, Odense, Denmark; ${ }^{2}$ Odense University Hospital, Department of Biochemistry and Pharmacology, Odense, Denmark; ${ }^{3}$ Odense University Hospital, Department of Immunology, Odense, Denmark; ${ }^{4}$ Odense University Hospital - Svendborg, Department of internal medicin - Section of Rheumatology, Svendborg, Denmark

Background: Fibrocytes are circulating cells with both myeloid and haemopoietic properties. They home in tissues with active inflammation, where they differentiate into mature fibrocytes that are involved in several inflammatory pathways. One complication of Rheumatoid Arthritis (RA) is Interstitial Lung Disease (ILD) resulting in high mortality and with limited treatment options. Fibrocyte levels are elevated in RA patients compared with healthy individuals and further increased in RA patients with signs of ILD (reduced diffusion capacity) and in patients with idiopathic lung fibrosis (IPF) or scleroderma. Thus, fibrocytes have been proposed as a future treatment target.

Objectives: We investigate the effect of corticosteroids, conventional Disease Modifying Anti Rheumatic Drugs (cDMARDs) and biological DMARDs (bDMARDs) on the in vitro differentiation of isolated peripheral blood mononuclear cells (PBMCs) into mature fibrocytes.

Methods: 10 participants were included (five patients with RA and five healthy controls). Information on current medication, sex, age, serology and disease activity were collected. PBMCS were isolated and cultured for 5 days in four wells per drug. Drugs included prednisolone, CDMARD (Methotrexate, Sulfasalasine, Hydroxycloroquine) and bDMARD (Inflectra, Etanecept, Tocilizumab, Adalimumab, Abatacept, Rituximab), and control wells with no drugs.

Results: Overall, abatacept and prednisolone significantly suppressed differentiation of PBMC into fibrocytes compared to control wells, see Figure $1(p=0.02$ and $p<0.01$, respectively) $(n=10)$. The reductive effect of Abatacept was significant among RA patients ( $p=0.009$ and) but not among healthy subjects. In overall analysis $(n=10)$, Abatacept reduced fibrocyte levels with an average of $44 \%$ overall and in the RA group $71 \%$ compared to control wells. Tocilizumab reduced the fibrocyte count with $63 \%$ overall and $45 \%$ in the RA group, although not significant $(p=0.07$ and $\mathrm{p}=0.06$ respectively)

Conclusion: Abatacept and prednisolon suppress the differentiation of mononuclear cells to mature fibrocytes in vitro in RA patients and data indicating a similar effect of Tocilizumab. Prednisolone are used in the treatment of RA-ILD but has a marked toxicity, so new treatment modalities are desirable. Our findings are in line with the fact that fibrocytes have the receptors targeted by abatacept, furthermore recent scleroderma research has shown Abatacept to reduce fibrocyte levels in vitro and Tocilizumab to potentially reduce lung and skin affection $(1,2)$ Further research using abatacept and tocilizumab to target fibrocytes are needed in order investigate the treatment potential of these drugs in RA-ILD. 

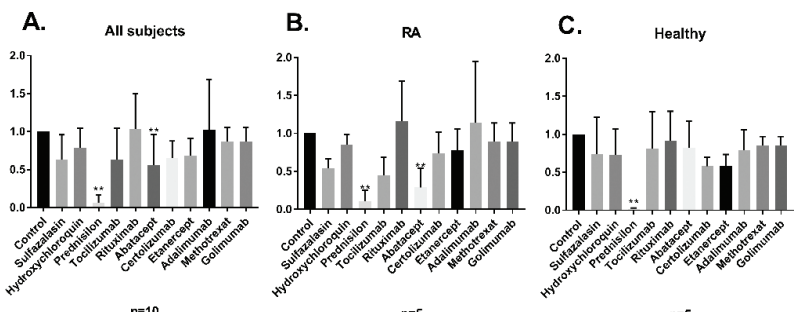

Figure 1. Effect of csDMARD, bDMARD and prednisolon on fibrocyte differentiation.

Ledgend: Demonstrates the different medical agents used and the number of cultured fibrocytes counted after 5 days of incubation. Values are mean +/- SEM. ** indicates statistical significance compared with the control well by ANOVA compared to the control $(p<0.05)$

\section{REFERENCES}

[1] Cutolo M, et al. Effects of CTLA4-Ig treatment on circulating fibrocytes and skin fibroblasts from the same systemic sclerosis patients: an in vitro assay. Arthritis Research \& Therapy. 2018;20.

[2] Khanna D, et al. Safety and efficacy of subcutaneous tocilizumab in systemic sclerosis: results from the open-label period of a phase II randomised controlled trial (faSScinate). Ann Rheum Dis. 2018;77(2):212-20.

Disclosure of Interests: None declared

DOI: 10.1136/annrheumdis-2019-eular.1915

\section{$\mathrm{AB} 0415$ \\ SURVIVAL OF BIOLOGIC AGENTS WITHIN A COHORT OF GREEK PATIENTS WITH RHEUMATOID ARTHRITIS. REAL WORLD DATA.}

Olga Katsouli ${ }^{1}$, Georgia Liantinioti ${ }^{1}$, Panagiota Katsouli ${ }^{1}$, Mary Pappa ${ }^{1}$, Markos Patsouras ${ }^{1}$, Theodoros Angelopoulos ${ }^{2}$, Sotiris Roussos ${ }^{3}$, VLACHOYIANNOPOULOS PANAYIOTIS ${ }^{1} .{ }^{1}$ Medical School National and Kapodistrian University of Athens, Department of Pathophysiology, Athens, Greece; ${ }^{2}$ Medical School National and Kapodistrian University of Athens, Athens, Greece; ${ }^{3}$ Medical School National and Kapodistrian University of Athens, Hygiene, Epidemiology and Medical Statistics, Athens, Greece

Background: Rheumatoid arthritis (RA) is treated successfully with biologic disease-modifying anti-rheumatic drugs (bDMARDs). However, a significant withdrawal rate, due to non-responsiveness or toxicity, remains a major barrier for their long-term use.

Objectives: To determine the withdrawal rate of the first bDMARD in a large cohort of RA patients due either, to non-responsiveness or toxicity. Methods: In this study, were included retrospectively 220 patients from our outpatient clinic. The following bDMARDs were evaluated: Etanercept ( $n=66$ patients), Adalimumab ( $n=61$ patients), Infliximab ( $n=70$ patients), Rituximab ( $\mathrm{n}=6$ patients) and others, such as Certolizumab pegol, Golimumab, Tocilizumab, Anakinra and Abatacept ( $\mathrm{n}=17$ patients). Disease activity was regularly measured by DAS-28 until the end of follow up. Kaplan-Meier plots were performed to examine the withdrawal rate of each biologic agent. Severity of AEs was classified according to the Common Terminology Criteria for Adverse Events (CTCAE, version 4.03). Results: A total of 220 patients (49 men, 171 women) were included in the analysis. The most frequently used first-line bDMARDs were Infliximab $(31.8 \%)$, Etanercept $(30 \%)$ and Adalimumab $(27.7 \%)$. The median of treatment duration with the first bDMARD was 15 months (Q1:8, Q3:40.5). Taking all bDMARDs as a whole, 126 patients $(57,27 \%)$ discontinued the first bDMARD, either due to $A E s(n=66,52.38 \%)$, or loss of efficacy $(n=60,47.62 \%)$ after a follow-up of 167 months. However the most withdrawals $(53.18 \%)$ occurred within the first 60 months of follow-up. Mean initial and final DAS-28 were: for those who discontinued treatment due to AEs $4.96 \pm 1.33(95 \% \mathrm{Cl} 4.64-5.28)$ and $3.43 \pm 1.47(95 \% \mathrm{Cl}, 3.08-3.79)$, respectively, for those who discontinued due to failure of therapy: 5.04 $\pm 1.37(95 \% \mathrm{Cl}, 4.70-5.39)$ and $4.91 \pm 1.27(95 \% \mathrm{Cl}, 4.59-5.23)$ respectively and for those who continue $4.79 \pm 1.55(95 \% \mathrm{Cl}, 4.47-5.10)$ and $2.63 \pm 1.06(95 \%$ $\mathrm{Cl}, 2.41-2.84)$, respectively.

Differences in survival among bDMARDs are attributed to their AEs and not to their efficacy. As far as the AEs are concerned, infections constituted the majority of cases $(n=23,34.8 \%)$, from which $34.8 \%$ needed hospitalization (CTCAE 3-4). The percentage of $12,1 \%$ of AEs is attributed to cancer cases, while allergic reactions counted for $31,8 \%$ of them. Survival of Etanercept within our cohort was significantly longer than those of other bDMARDs.
Conclusion: Biologic agents are important drugs in our armamentarium to combat RA. However nearly $50 \%$ of patients discontinue these agents after 60 months of treatment due either to AEs or inefficacy. Differences in survival of bDMARDs were associated not to their inefficacy, but to their AEs. Choosing the right drug for the right patient is a matter of advanced research involving a better understanding of the plasticity of the pathways of inflammation and specific biomarker selection.

\section{REFERENCES}

[1] C. Lampropoulos, P. Orfanos, M. Manoussakis, A. Tzioufas, H. Moutsopoulos, P. Vlachoyiannopoulos. Treat-to-target biologic therapy in patients with rheumatoid arthritis is more efficacious and safe compared to delayed initiation of biologics:A real-world study.ClinExpRheumatology.2017;35:192-200.

[2] C. Lampropoulos, P. Orfanos, V. Bournia, T. Karatsourakis, C. Mavragani, D. Pikazis, M. Manoussakis, A. Tzioufas, H. Moutsopoulos, P. Vlachoyiannopoulos.Adverse events and infections in patients with rheumatoid arthritis treated with conventional drugs or biologic agents: a real world study. ClinExpRheumatology.2015;33:216-224.
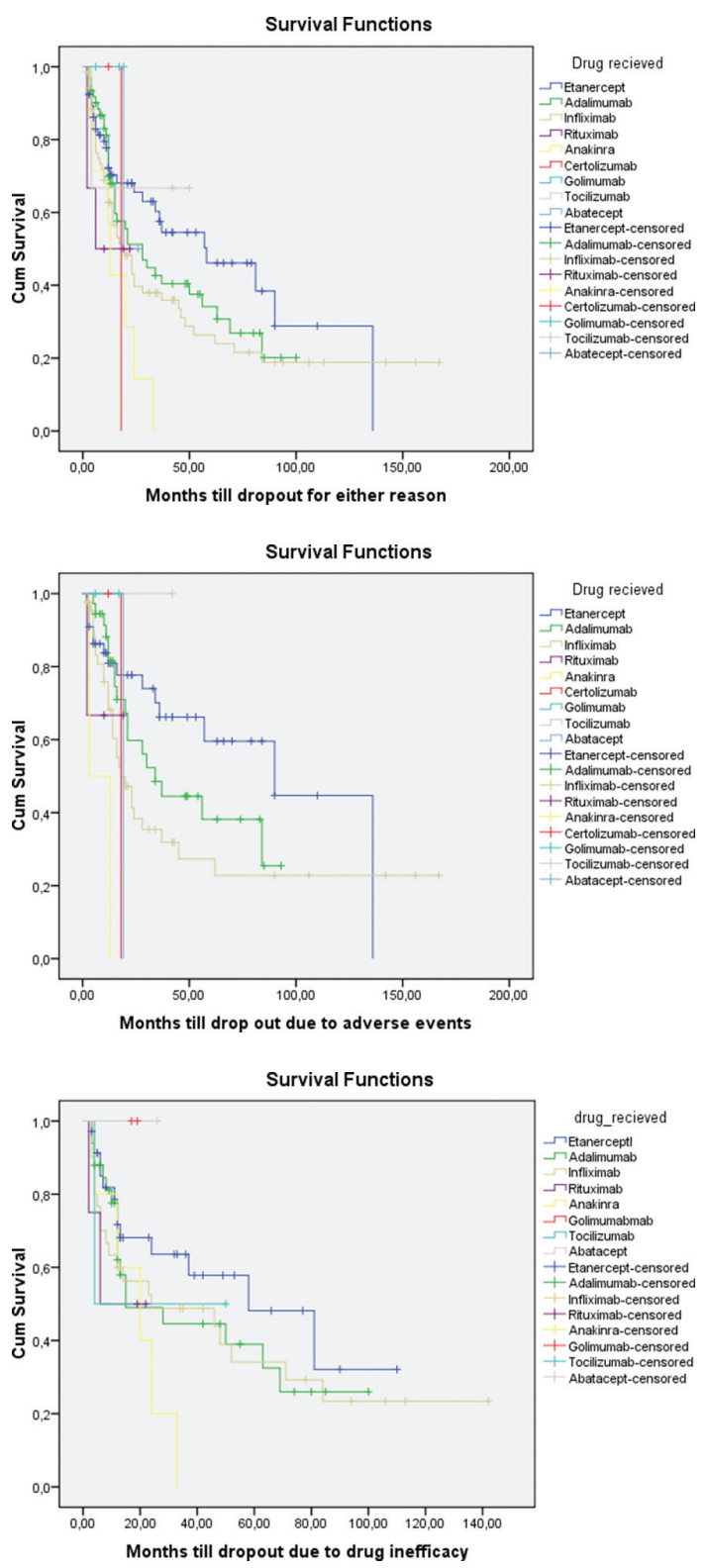

Disclosure of Interests: Olga Katsouli: None declared, Georgia Liantinioti: None declared, Panagiota Katsouli: None declared, MARY PAPPA Employee of: Bayer, Markos Patsouras: None declared, Theodoros Angelopoulos: None declared, Sotiris Roussos: None declared, PANAYIOTIS VLACHOYIANNOPOULOS: None declared DOI: 10.1136/annrheumdis-2019-eular.7308 